\title{
SEGMENTATION OF MAMMARY LESIONS IN ULTRASOUND IMAGES APPLYING MASK R-CNN
}

\author{
Claudia Raquel Ibarrola Chamorro \\ Latin American Center for Open Technologies \\ Foz do Iguacu, Brazil \\ Email: clauibarrola87@gmail.com
}

\author{
Wagner Coelho de Albuquerque Pereira \\ Biomedical Engineering Program / COPPE / UFRJ \\ Federal University of Rio de Janeiro \\ Rio de Janeiro, Brazil \\ Email:wcap58@gmail.com
}

\begin{abstract}
Breast cancer is the most frequent malignant tumor in women and one of the most common in the world. One of the most important issues in this condition is early detection. Computer-aided diagnostic (CAD) systems are objects of research, aiming to provide a second opinion to the health professional. A fundamental aspect within the CAD system is the segmentation of the lesion, allowing an adequate extraction of the lesion characteristics. The use of a computerized segmentation method helps eliminate human variability and, consequently, improve the performance of the lesion classifier. Convolutional Neural Networks (CNNs) are being used in segmentation problems, such as various types of medical imaging, people and road signs detection, for example. So inspired by these promissing results, the present work has as main objective to analyze and implement the Mask R-CNN as a tool of segmentation of mammary lesions in images obtained by ultrasound, to propose an efficient method of segmentation, aiding in the classification process of the CAD systems.
\end{abstract}

Keywords-segmentation; breast cancer; ultrasonography

\section{INTRODUCTION}

According to the International Agency for Research on Cancer (IARC), breast cancer is currently the most frequent neoplasm and the greatest cause of death among women in the world [1]. The causes of the onset of this type of cancer are multifactorial, covering external causes such as the environment and habits of the patient, as well as internal causes, for example, a genetic predisposition [2]. Because of this large number of influences, early detection is one of the safest ways to combat the disease, because the earlier the cancer is detected, the greater the effectiveness of the treatment adopted and, consequently, the greater the patient's chances of cure [3].

Mammography has been considered as the only technique of imagem that contributed, through a periodic monitoring program, to a reduction of mortality due to breast cancer. Mammograms can usually detect this type of cancer in its early stages [4]. A second method for breast cancer screening is ultrasonography [5], which is portable, easy handling and nonionizing technique. Nevertheless, image formation includes the generation of speckle noise, which leaves the image with grainy appearance, making it difficult the separability between the objects. The correct interpretation of ultrasound images is a critical stage in diagnosis. Normally, this interpretation is done visually by a radiologist, which analyzes the image and provides the diagnosis to the patient [6]. In this context, auxiliary diagnostic systems, also called CAD (Computer-Aided Diagnosis) systems, are computational aids for the diagnosis of injuries, which are developed to evaluate and assist in the interpretation of images [7]. This type of system has been increasingly used in the analysis of medical images, since it is possible to find applications related to the detection of throat cancer, cardiac problems and also in mammary cancer [8]. These systems are usually composed of a set of steps, ranging from the acquisition of the image to the classification of the lesion in benign or malignant [7].

A fundamental aspect within a CAD system is the segmentation of the lesion image, since the morphometric and ecotexture characteristics are used to quantify the lesions [9]. There are cases where segmentation is performed manually by radiologists, outlining the contour of the lesion. However, this procedure is subjective, since there is inter- and intraobserver variability in the interpretation of the image [10]. Fully automatic targeting methods are preferred today [11], [13] because operator dependency is virtually eliminated, leading to fully automated CAD systems [12]. This work proposes the application of a convolutional neuronal network called Mask R-CNN, with the purpose of automating the segmentation of ultrasound images in a CAD system and provide the health professional with a tool that acts as a second opinion in the disease diagnosis process.

\section{Material AND METHODS}

It will denoted the database and tools used in the image segmentation procedure.

\section{A. Image Database}

The database of images used belongs to the Biomedical Engineering Program of COPPE / UFRJ, obtained through standard tests performed on patients in the diagnostic process. There are 413 benign lesions and 228 malignant 
lesions, all confirmed by biopsy. Each image was manually segmented by a medical specialist. The images were acquired with different setups by several specialists and equipment

\section{B. Image Annotation}

Images were annotated using (VGG Image Annotator - v 1.0.6), which is a tool that can be used to define regions in an image and create textual descriptions of those regions [14]. The annotations were made in polygon format and later exported in a .json file.

\section{Mask R-CNN}

The implementation of Mask R-CNN [16] was done using the programming language python version 3.6, the open source neural network library written in Python, Keras and the open source library for machine learning, TensorFlow. The implemented model generates bounding boxes and targeting masks for each instance of an object in the image. Mask R-CNN, extends Faster R-CNN [15] by adding a branch for predicting segmentation masks on each Region of Interest (ROI) [16].

\section{RESULTS}

The preliminary evaluation was done by averaging the IoU (Intersection over Union) in three groups of random images with different amounts. IoU also called the Jaccard index, is essentially a method for quantifying the overlap percentage between the destination mask and our forecast output. The IoU metric measures the number of common pixels between the destination and predictive masks divided by the total number of pixels present in the two masks. And it is defined by the equation 1 [17].

$$
I o U=\frac{\text { area of overlap }}{\text { area of union }}
$$

Both tests presented in this material were made in images of benign lesions included in the database mentioned in II-A. To test the model in a first step two datasets from the main database were created. For the first dataset, 60 images were randomly selected, of which 48 are for training and 12 images for validation. In the following step a second dataset called dataset 2 was used, containing 330 for training and 83 images for validation. In this section we explain the results obtained in the various processes in individual images, the figures correspond to the result of the second dataset. Figure 1 show on the left two ultrasound images with breast lesion and on the right the result of the segmentation mask in the training phase. In figure 2, 10 regions of interest of the 200 that were generated in the training process are shown. Of the 200 ROIs, 52 are classified as positive ROIs (they are part of the lesion) and 148 as negative ROIs (they are part of the background). And in figure 3 we have the final proposed segmentation mask generated for the example image (left) and the and the image segmented manually (right).
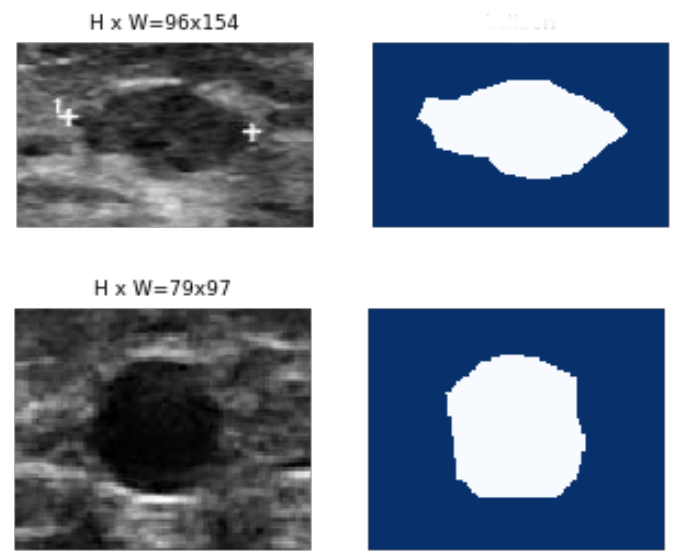

Figure 1. Masks generated for the training phase. On the left two ultrasound images with breast lesion and on the right the result of the segmentation mask generated in the training phase for each image.

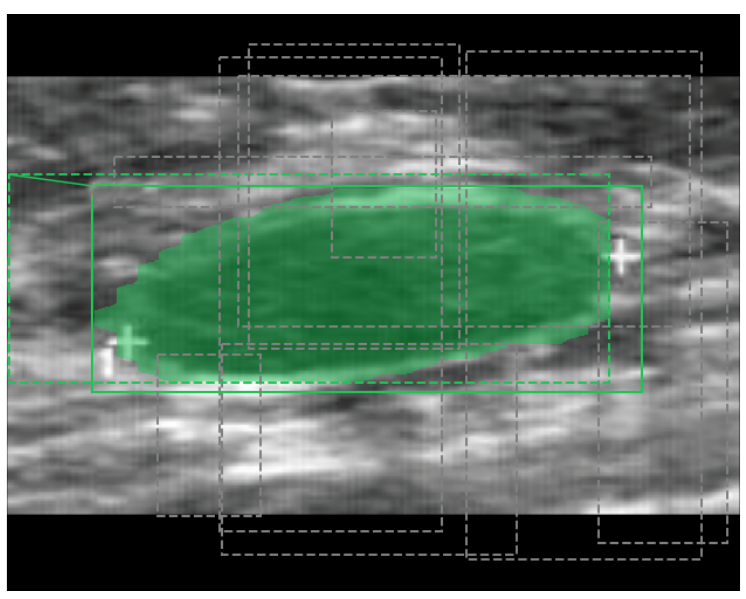

Figure 2. Region of interest and bounding boxes. 10 regions of interest of the 200 that were generated in the training process.

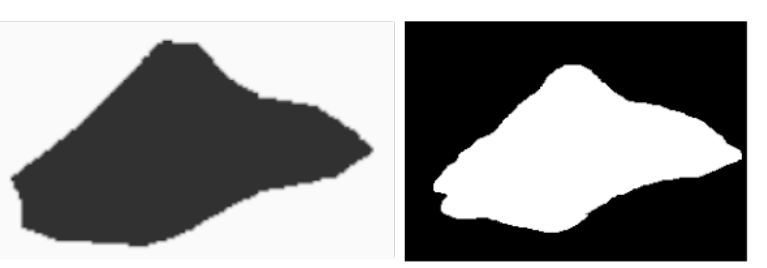

Figure 3. Resultant targeting mask. The final proposed segmentation mask generated for the example image (left) and the and the image segmented manually (right). 


\begin{tabular}{|l|l|l|}
\cline { 2 - 3 } \multicolumn{1}{c|}{} & 10 images & 50 images \\
\hline dataset1 & $0.84 \pm 0.032$ & $0.79 \pm 0.04$ \\
\hline dataset2 & $1.0 \pm 0.00$ & $0.96 \pm 0.038$ \\
\hline
\end{tabular}

Table I

IoU average and Standard Deviation for image segmentation with two sets of test images

Table I shows the level of success achieved in the first tests conducted with the Mask-RCNN, the values go from 0 to 1 , where 0 equals no success and 1 represents $100 \%$ success, as well as the average deviation presented in each group of tests.

It is observed that the accuracy of the model presents a considerable difference depending on the datasets, in this point it is important to emphasize that the dataset 1 contains a considerably smaller number of images in relation to the dataset2, which makes the model present a better performance with the one that has more learning options.

\section{Discussion AND CONClusion}

In the processing of ultrasound images for CAD systems, obtaining a good segmentation is a crucial task. In the first experiments carried out with the implementation of the Mask $\mathrm{R}-\mathrm{CNN}$ in a part of our database we obtained a high degree of acertivity in the segmentation proposals made by the network.

For the next steps, we intend to continue with the execution of the R-CNN mask and establish a neuronal model to provide greater precision in terms of segmentation and detection of lesions in medical images. To achieve this objective the following intended future activities are:

- Perform detection and segmentation of malignant lesions in the images contained in the database.

- Conduct experiments with a more robust database.

- Evaluate the results obtained using other recommended metrics in literature.

\section{ACKNOWLEDGMENT}

\section{CNPq 311.650/2017-1, FAPERJ E-26/203.041/2015, CAPES/PROEX}

\section{REFERENCES}

[1] J. Ferlay, M. Colombet, I. Soerjomataram, C. Mathers, DM. Parkin, M. Piñeros, A. Znaor, and F. Bray, Estimating the global cancer incidence and mortality in 2018: GLOBOCAN sources and methods. International journal of cancer, 2019.

[2] RA. Smith, KS. Andrews, D. Brooks, SA. Fedewa, D. Manassaram-Baptiste, D. Saslow, OW. Brawley and RC. Wender, Cancer screening in the United States, 2017: a review of current American Cancer Society guidelines and current issues in cancer screening. CA: a cancer journal for clinicians, 2017.

[3] M. Giger Computer-aided diagnosis of breast lesions in medical images. Computing in Science \& Engineering, 2000.
[4] L. Tabr, B. Vitak, T. Chen, A. Yen, A. Cohen, T. Tot, S. Chiu, S. Chen, J. Fann, J. Rosell, H. Fohlin, R. Smith, S. Duffy, Swedish Two-County Trial: Impact of Mammographic Screening on Breast Cancer Mortality during 3 Decades. Radiology:Imaging Cancer, 2011, https://doi.org/10.1148/radiol.11110469

[5] K. Kazemi, A. Adibi, M. Golshahi and M. Sirus, Breast cancer screening: Evidence of the effect of adjunct ultrasound screening in women with unilateral mammography-negative dense breasts. J Res Med Sci. 2015 Mar; 20(3): 228232.

[6] G. Albonico, Seleo de Atributos e Classificao Automtica de Leses Mamrias em Imagens de Ultrassom. Dissertation (Master degree) - State University of Western Paran, Brazil, 2017

[7] W. Flores, Desarrollo de una Metodologa Computacional para la Clasificacin de Lesiones de Mama en Imgenes Ultrasnicas. Thesis (Doctorate) - Center for Research and Advanced Studies, National Polytechnic Institute. Mexico, 2009

[8] W. Flores, W. Pereira and A. Catelli, Improving classification performance of breast lesions on ultrasonography. Pattern Recognition, Elsevier, 2015.

[9] J. Rogowska, Overview and fundamentals of medical image segmentation. Handbook of Medical Imaging Processing and Analysis, Academic Press, San Diego, 2000

[10] M. Calas, R. Almeida, B. Gutfilen and W. Pereira, Interobserver concordance in the BI-RADS classification of breast ultrasound exams. Clinics, SciELO Brasil, 2012.

[11] RM. Menchon and JL. Sancho, Fully automatic segmentation of ultrasound common carotid artery images based on machine learning. Neurocomputing, 2015.

[12] M. Xian, Y. Zhang and H. Cheng, Fully automatic segmentation of breast ultrasound images based on breast characteristics in space and frequency domains. Pattern Recognition, Elsevier, 2015.

[13] Y. Li, Segmentation of Medical Ultrasound Images Using Convolutional Neural Networks with Noisy Activating Functions You. Stanford Edu, 2016

[14] A. Dutta, A. Gupta and A. Zissermann. VGG Image Annotator (VIA), 2016. http://www.robots.ox.ac.uk/ vgg/software/via/

[15] S. Ren, K. He, R. Girshick, and J. Sun, Faster R-CNN: To- wards real-time object detection with region proposal networks. NIPS, 2015.

[16] K. He, R. Gkioxari, G. Dollar and P. Girshick, Mask R-CNN. Proceedings of the IEEE International Conference on Computer Vision, 2017. https://doi.org/10.1109/ICCV.2017.322

[17] A. Rahman and Y. Wang, Advances in Visual Computing, 2013. https://doi.org/10.1007/978-3-642-41939-3 